\title{
Psicooncología
}

ISSN: 1696-7240

\section{Beneficios de la musicoterapia como opción integrativa en el tratamien- to oncológico}

\author{
Jaime Carrasco García ${ }^{1}$, Ignacio González López², Ana Belén Cañizares Sevilla ${ }^{3}$
}

Recibido: 15 de abril de 2020 / Aceptado: 20 de agosto de 2020

Resumen: Objetivo: Identificar la eficacia de los tratamientos musicoterapéuticos que aborden la sintomatología en pacientes con cáncer y que constaten algún tipo de beneficio psicológico, fisiológico, espiritual, social o intelectual. Método: Se realizó un análisis documental que supuso un estudio comparativo de evidencias científicas. Resultados: La musicoterapia minimiza los efectos fisiológicos de tratamientos específicos oncológicos como la quimioterapia, se usa como complemento de la morfina o el sufentanilo disminuyendo el dolor e influye en la vida emocional del paciente. Conclusiones: Es una disciplina que demuestra evidencias positivas en situaciones de tipo emocional reduciendo la depresión, la ansiedad y el estrés, ayudando en la relajación y mejorando el umbral del dolor, postulándose como una opción no-farmacológica que debiera conducir a una implantación cada vez más normalizada en los centros hospitalarios.

Palabras clave: Musicoterapia, cáncer, bienestar, depresión, ansiedad, estrés.

\section{[en] Benefits of music therapy as an integrative option in oncological treatment}

\begin{abstract}
Objective: To identify the efficacy of music therapy treatments that address symptoms in cancer patients and show some type of psychological, physiological, spiritual, social or intellectual benefit. Method: A documentary analysis was carried out that involved a comparative study of scientific evidence. Results: Music therapy minimizes the physiological effects of specific oncological treatments such as chemotherapy, it is used as a complement to morphine or sufentanil, reducing pain and influencing the emotional life of the patient. Conclusions: It is a discipline that shows positive evidence in emotional situations reducing depression, anxiety and stress, helping in relaxation and improving the pain threshold, postulating itself as a non-pharmacological option that should lead to an implantation every time. more standardized in hospital centers.
\end{abstract}

Keywords: Music therapy, cancer, wellness, depression, anxiety, stress.

1 Jaime Carrasco García. Facultad de Ciencias de la Educación y Psicología. Universidad de Córdoba, España.

E-mail: jaime.carrasco.gar@gmail.com

2 Ignacio González López. Facultad de Ciencias de la Educación y Psicología. Universidad de Córdoba, España. E-mail: ed1goloi@uco.es

3 Ana Belén Cañizares Sevilla. Facultad de Ciencias de la Educación y Psicología. Universidad de Córdoba, España.

E-mail: eo1casea@uco.es

* Dirección de correspondencia: Jaime Carrasco García. Facultad de Ciencias de la Educación y Psicología; Departamento de Educación, Área de Métodos de Investigación y Diagnóstico en Educación. Avda. San Alberto Magno, s/n - 14071 Córdoba (España).E-mail: jaime.carrasco.gar@gmail.com; m62cargj@uco.es 
Sumario. 1. Introducción 1.1. Musicoterapia como complemento terapéutico 2. Método 3. Análisis de datos 4. Resultados 5. Conclusiones 6. Referencias bibliográficas

Cómo citar: Carrasco García J, González López I, Cañizares Sevilla AB. Beneficios de la musicoterapia como opción integrativa en el tratamiento oncológico. Psicooncología 2020; 17: 335-355. doi: 10.5209/ psic.68812

\section{Introducción}

La medicina integrativa es un método de atención globalizado que incluye la mente, el cuerpo y el espíritu del paciente. Martí et al. (2015a) ${ }^{(1)}$ argumentan que combina la medicina convencional con prácticas de la medicina complementaria y otras alternativas que han sido más prometedoras, como la Musicoterapia. En la misma línea se pronuncian De Prados et al. (2003) ${ }^{(2)}$ situando la Musicoterapia entre las terapias complementarias que se están incorporando al tratamiento de los pacientes oncológicos por favorecer su bienestar emocional, y aplicar de manera sistemática la música con una intencionalidad terapéutica para reducir la depresión, la ansiedad, el estrés y el dolor. Por otra parte, Brad et al. (2016) ${ }^{(3)}$ han encontrado que las intervenciones de musicoterapia y medicina musical se han utilizado para aliviar los síntomas y los efectos secundarios del tratamiento y abordar las necesidades psicosociales en personas con cáncer.

Quiroga et al. (2016) $)^{(4)}$ señalan que la oncología integrativa suplementa el tratamiento convencional con las terapias complementarias que evidencian beneficios. Las llamadas actividades mente-cuerpo-espíritu, entre las que se encuentra la Musicoterapia, juegan un papel importante a la hora de disminuir los síntomas, aumentar el bienestar y mejorar la calidad de vida de los pacientes oncológicos durante el tratamiento médico convencional.

En la actualidad, la Musicoterapia se inserta con más presencia en el campo oncológico, se realiza en un momento muy preciso de la enfermedad y dentro de un programa específico de la atención de psicooncología, dado que ambas presentan rasgos terapéuticos comunes ${ }^{(5)}$. Así, proyectos como el del Hospital Infantil Miguel Servet de Zaragoza ${ }^{(6)}$ y del Hospital Cruces de Bilbao ${ }^{(7)}$, buscan mejorar la calidad de vida de niños y niñas con cáncer, reduciendo los niveles de ansiedad, estrés y dolor y favoreciendo el buen estado de ánimo a través de la improvisación musical terapéutica, por medio de la música en vivo (cantada o sonorizada) de cuentos musicales. Se constatan cambios significativos relativos a cómo la presencia de la música ha beneficiado en la reducción de situaciones de estrés y el aumento de la relajación, siendo más notables en los momentos de intervención de técnicas de enfermería y procedimientos médicos.

\subsection{Musicoterapia como complemento terapéutico}

El cáncer es un problema de salud mundial que sigue creciendo, por ello todos los países actúan para mejorar su prevención. Por un lado, con pruebas de detección precoz, tratamientos y cuidados médicos específicos y, de otro, a través de la medicina complementaria y alternativa en la que se utilizan prácticas que se considera no pertenecen a la atención médica convencional. En este contexto, se pone de manifiesto 
la influencia de la música cuando se utiliza con una intencionalidad terapéutica sobre la mejora en la calidad de vida de las personas por su influencia en el bienestar tanto físico como psicológico ${ }^{(8)}$.

La Musicoterapia es un valor en alza, que cobra presencia en todo el mundo gracias a las investigaciones realizadas que avalan la evidencia de la influencia de la música, usada con intención terapéutica, sobre el bienestar psicológico, social y emocional y sobre la calidad de vida de las personas que están sanas satisfaciendo, además, las necesidades de niños y adultos con discapacidades o enfermedades. Ante la preocupación de dar una solución no-farmacológica a los usuarios/pacientes y a sus cuidadores que les ayude a afrontar positivamente el trance en que se encuentran se está empleando, cada vez más, la musicoterapia con rigurosidad científica y metodológica ${ }^{(9)}$.

La Musicoterapia moderna aparece en EEUU a principios del S. XX, cuando los músicos entretenían en los hospitales a pacientes que sufrían traumas físicos y emocionales de las guerras, y se observó que facilitaba su recuperación. En la Universidad de Columbia, la enfermera Margaret Anderton impartió cursos de musicoterapia (1919) y fundó la Clínica Anderton, que incluía los servicios de música para la recuperación de los soldados canadienses heridos durante la Primera Guerra Mundial ${ }^{(10)}$. Desde entonces, los estudios de investigación en Musicoterapia han proliferado y, gracias a los resultados de los autores plasmados en las revistas y las bases de datos, se facilita el acceso a la información para estar al día sobre las últimas tendencias.

Miller, Sternheimer, Clynes y Golstein, citados en Poch (2002) ${ }^{(11)}$, sostienen que la música produce en nuestro organismo efectos bioquímicos positivos o negativos, dependiendo del tipo de música usada, ya que algunas frecuencias vibratorias de los sonidos (notas musicales) estimulan los aminoácidos de cierta proteína. Así, la música sedante puede estimular la liberación de hormonas, las cuales a su vez actúan sobre receptores específicos del cerebro y sobre neurotransmisores, que pueden llevar a aliviar el dolor. Efectivamente, favorece la producción de hormonas (endorfinas, dopamina, acetilcolina y oxitocina) que actúan como neurotransmisores opiáceos producidos en el Sistema Nervioso Central, lo que provoca bienestar en las personas. En la misma línea, Lacárcel (2003) indica que:

"La música afecta de tal forma al nivel psicofisiológico y emocional de la persona que existe una necesidad de estimular el pensamiento positivo y las nociones constructivas mediante la música debido a que estas modifican las endorfinas y las células $\mathrm{C}$ del organismo, que forman parte del sistema inmunológico"(12).

Otros autores observan beneficios psicológicos sobre el papel potencial de la música para ayudar a abordar y articular estados emocionales complejos, como los sentimientos que los pacientes de cáncer pueden experimentar durante la evolución de su enfermedad o un proceso de duelo ${ }^{(13)}$.

En los adultos, la musicoterapia se puede utilizar como un instrumento de cambio en el estado de ánimo e influir de manera rápida en sus emociones, y por ende en su calidad de vida. En el área afectivo-emocional, Rickson y Gold, citados en Benedito $(2010)^{(14)}$, observaron resultados positivos disminuyendo la tensión y mejorando la 
autoestima; en el área de la comunicación, incrementando la capacidad de expresarse y la creatividad; en el área social, aumentando la conciencia social; en el área cognitiva, incrementando la atención, aprendizaje y memoria reciente; en el área fisiológica, produciendo efectos similares al éxtasis y otro tipo de drogas en nuestro cerebro. En este mismo sentido se pronuncian Oslé $(2011)^{(15)}$, Ribeiro et al. $(2018)^{(16)}$, Fernández et al. (2018) $)^{(17)}$ y Marins et al. $(2018)^{(18)}$.

Hay autores que, en sus investigaciones, han encontrado beneficios diversos. En primer lugar, beneficios sociales en ancianos sometidos a sesiones de musicoterapia (19); mejoras en las habilidades comunicativas en niños con Trastornos del espectro autista (TEA) ${ }^{(20)}$, como las llevadas a cabo por Gattino et al. (2016) ${ }^{(21)}$, Marom et al. (2018) ${ }^{(22)}$, o Bergmann et al. (2016) ${ }^{(23)}$; y Valdés et al. (2012) ${ }^{(24)}$ en habilidades de concentración y de comportamiento social en niños con Trastorno por déficit de atención con hiperactividad (TDAH).

En segundo lugar, se encuentran los beneficios fisiológicos. Facilita el mejor conocimiento de sí mismo a aquellos pacientes que tienen perdida o limitada su orientación temporal ${ }^{(25)}$ a través del ritmo. Produce efectos relajantes al liberar dopamina, que genera respuestas de reforzamiento positivo, siguiendo las mismas pautas que la fisiopatología de las conductas adictivas y del consumo de sustancias psicoactivas $^{(26)}$. Reduce el dolor y produce relajación autógena ${ }^{(27)}$.

En tercer lugar, confiere beneficios psicosociales. Puede ayudar a las víctimas de traumas ${ }^{(28)}$. Contribuye a minimizar los efectos negativos propios del proceso de envejecimiento, reforzando la capacidad memorística, mejora las habilidades motoras y los lenguajes expresivo y receptivo ${ }^{(29)}$. Produce retraso de la aparición del vómito y/o náusea ${ }^{(30)}$.

Otros autores refieren beneficios intelectuales en la memoria de pacientes con enfermedad de Alzheimer ${ }^{(31)}$, así como mejora en la depresión y facultades intelectuales $^{(32,33)}$.

Por último, hay que destacar los beneficios espirituales, como apunta Foster $(2010)^{(34)}$, porque la musicoterapia llega de manera efectiva al dolor y al sufrimiento de la persona moribunda consiguiendo paz y tranquilidad. Produce también consuelo espiritual en familiares de fallecidos por cáncer ${ }^{(35)}$.

\section{Método}

El objetivo de este estudio consiste en identificar la eficacia de los tratamientos musicoterapéuticos que aborden la sintomatología en pacientes con cáncer y que constaten algún tipo de beneficio psicológico, fisiológico, espiritual, social o intelectual. De esta manera se evidenciará la importancia que tiene la Musicoterapia en la calidad de vida y salud percibida por los pacientes aquejados de esta enfermedad.

Para ello, se llevó a cabo un análisis documental, entendido como un proceso dinámico que consiste en la recogida, clasificación, recuperación y distribución de la información a partir de artículos científicos ${ }^{(36)}$. Para su desarrollo se establecieron tres fases:

En una primera fase, se buscaron artículos publicados entre los años 2014 a 2019 en las aplicaciones ProQuest y EBSCOhost web, seleccionando aquellos que cumplían los siguientes criterios: ensayos clínicos con intervención de Musicoterapia/diseño experimental en pacientes con cáncer, "MusicTherapy and cancer" y si estaban o no "evaluados por pares". 
En una segunda fase, como garantía de calidad, se localizó el factor de impacto en el campo de investigación de las revistas donde aparecen publicados los artículos encontrados. Para su consulta, se ha utilizado el Journal Citation Report (JCR), que ofrece el indicador de calidad más valorado por los organismos de evaluación de la actividad investigadora y que mide el impacto de una revista en función de las citas recibidas por los artículos publicados y recogidos en la Web of Science (WOS); y Scimago Journal Rank (SJR), que calcula el factor de impacto basándose en la información incluida en la base de datos SCOPUS.

En una tercera fase, a través de los textos completos o los resúmenes proporcionados por las bases de datos consultadas, se revisaron los artículos seleccionados, según las unidades de análisis establecidas: estrategia de intervención de musicoterapia/ diseño, muestra, características del grupo experimental, características del grupo control y resultados obtenidos, con el objeto de testar la efectividad de las diferentes intervenciones musicoterapéuticas sobre la respuesta emocional de los pacientes.

\section{Análisis de datos}

Tras la búsqueda bibliográfica realizada, con la primera condición de aceptar que fuesen artículos publicados en español o en inglés, durante el período 2014 al 2019, se obtuvieron 404 referencias en EBSCO y 44 referencias en ProQuest. De ellas, se escogieron aquellos trabajos publicados en revistas con factor de impacto JCR y/o SJR, reduciéndose dicho número. De éstos, se excluyeron los que no devolvieron ni texto completo ni resumen, así como los repetidos en ambas aplicaciones, y de los resultantes se descartaron aquellos que no cumplieron los criterios de inclusión propuestos para el estudio: ensayos clínicos con intervención de Musicoterapia/ diseño experimental con pacientes con cáncer, obteniéndose un total de 17 artículos pertenecientes a 9 revistas (ver Tabla 1).

Tabla 1. Nombre de la publicación y factor de impacto de los artículos analizados.

\begin{tabular}{|c|c|c|c|c|}
\hline & Nombre de la revista & $\begin{array}{l}\text { Factor de } \\
\text { Impacto JCR } \\
\text { (año 2019) }\end{array}$ & $\begin{array}{l}\text { Factor de } \\
\text { Impacto SJR } \\
\text { (año 2019) }\end{array}$ & $\begin{array}{c}\mathrm{N}^{\mathrm{o}} \\
\text { Artículos } \\
\text { por revista }\end{array}$ \\
\hline 1 & $\begin{array}{l}\text { Deutsches Arzteblatt } \\
\text { International (Alemania) }\end{array}$ & Q1 (4,796) & Q3 $(0,429)$ & 1 \\
\hline 2 & $\begin{array}{c}\text { European Journal of Oncology } \\
\text { Nursing (USA) }\end{array}$ & Q1 $(1,876)$ & Q2 $(0,753)$ & 1 \\
\hline 3 & $\begin{array}{l}\text { Journal of Alternative and } \\
\text { Complementary Medicine } \\
\text { (USA) }\end{array}$ & Q2 $(2,109)$ & Q1 $(0,556)$ & 1 \\
\hline 4 & $\begin{array}{c}\text { Oncology Nursing Forum } \\
\text { (USA) }\end{array}$ & Q2 (1,728) & Q3 $(0,588)$ & 1 \\
\hline
\end{tabular}




\begin{tabular}{|c|c|c|c|c|}
\hline & Nombre de la revista & $\begin{array}{c}\text { Factor de } \\
\text { Impacto JCR } \\
\text { (año 2019) }\end{array}$ & $\begin{array}{c}\text { Factor de } \\
\text { Impacto SJR } \\
\text { (año 2019) }\end{array}$ & $\begin{array}{c}\mathrm{N}^{\mathrm{o}} \\
\text { Artículos } \\
\text { por revista }\end{array}$ \\
\hline 5 & $\begin{array}{c}\text { Supportive Care in Cancer } \\
\text { (USA) }\end{array}$ & Q3 $(2,635)$ & Q2 $(1,062)$ & 2 \\
\hline 6 & $\begin{array}{l}\text { Journal of Music Therapy } \\
\text { (USA) }\end{array}$ & Q3 $(1,206)$ & Q1 $(0,749)$ & 2 \\
\hline 7 & $\begin{array}{l}\text { Nordic Journal of Music } \\
\text { Therapy (Noruega) }\end{array}$ & Q4 $(0,913)$ & Q2 $(0,454)$ & 4 \\
\hline 8 & $\begin{array}{l}\text { Indian Journal of Palliative Care } \\
\text { (India) }\end{array}$ & - & Q3 $(0,327)$ & 2 \\
\hline 9 & Revista Psicooncología (España) & - & Q4 $(0,184)$ & 3 \\
\hline
\end{tabular}

Se describe aquí el factor de impacto de las revistas encontradas. Todas poseen indicador JCR y SJR, excepto dos, Indian Journal of Palliative Care y Psicooncología, que se encuentran únicamente indizadas en SCOPUS. Las más recurrentes y que recogen evidencias empíricas sobre el objeto de estudio son las editadas en Estados Unidos con 5 revistas ( 7 artículos), Noruega, con 1 revista (4 artículos) y España, con 1 revista ( 3 artículos). En menor medida aparecen experiencias en revistas médicas de India ( 2 artículos) y Alemania (1 artículo).

Los 17 artículos seleccionados fueron sometidos a un análisis de contenido siguiendo las premisas propuestas en la fase 3 .

A continuación, se presentan los datos organizados en torno a los tipos de beneficios obtenidos en cada uno de los artículos seleccionados y según sean de carácter psicológico, fisiológico, espiritual, social o intelectual, con un análisis sobre la efectividad de las diferentes intervenciones musicoterapéuticas, siguiendo las unidades de análisis propuestas en esta fase.

Los artículos analizados presentan heterogeneidad en cuanto a los beneficios que produce la intervención: dos demuestran beneficios psicológicos y fisiológicos; diez psicológicos; tres fisiológicos; y, por último, uno intelectuales y uno espirituales. Siguiendo este orden, se muestran los beneficios referidos (tablas 2, 3, 4, 5 y 6). Todos utilizan un diseño empírico experimental, 8 de ellos con grupos experimental y control $(47,05 \%)$ y 9 solamente con grupo experimental $(52,94 \%)$.

En la Tabla 2 se exponen los tres artículos que refieren beneficios psicológicos y fisiológicos con los pacientes. El primero, con adultos alemanes con cáncer en cuidados paliativos que, tras la intervención, los pacientes del grupo experimental refieren mejoras en el bienestar y en la relajación (en relación con el grupo control). El segundo, con mujeres adultas estadounidenses con cáncer de mama que reciben quimioterapia y que, tras la intervención, manifiestan una mejora significativa en el estado de ánimo y una disminución significativa del estado de fatiga (no hay grupo control). El tercero, adultos estadounidenses con cáncer y que tras la intervención mejoran el estado de ánimo, ansiedad, relajación y dolor que dependen de la motivación de los pacientes sobre su futuro. 


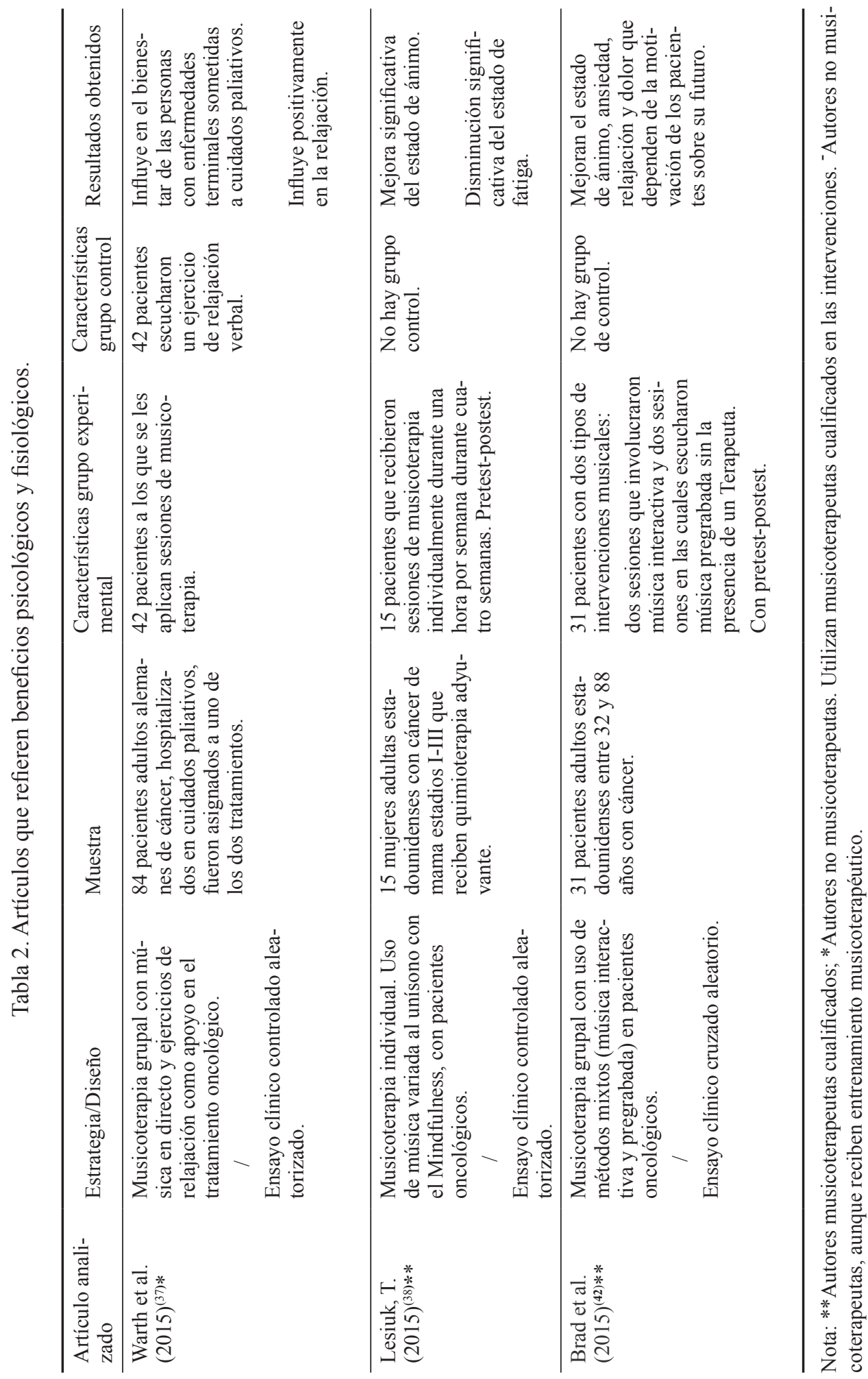




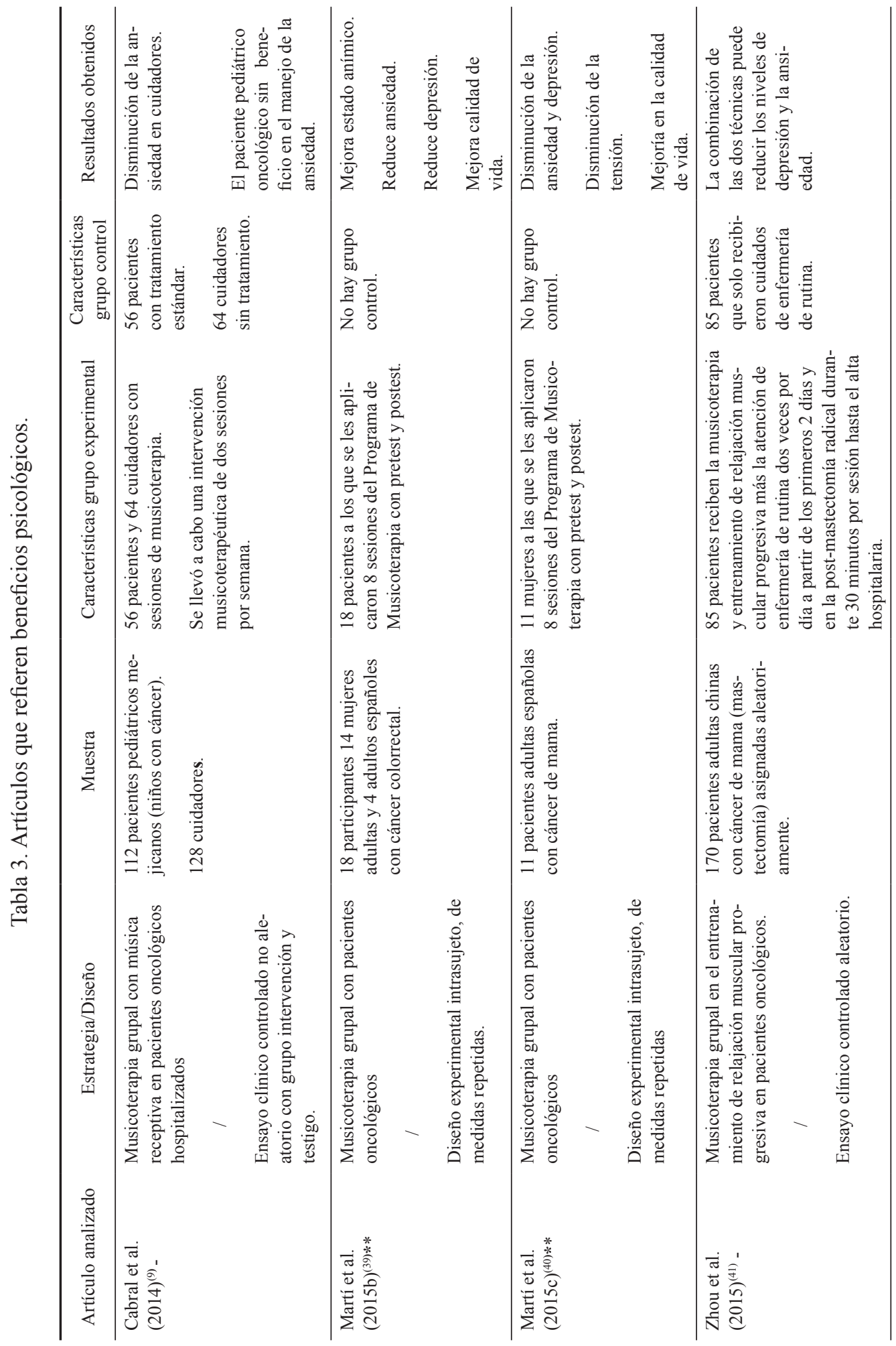




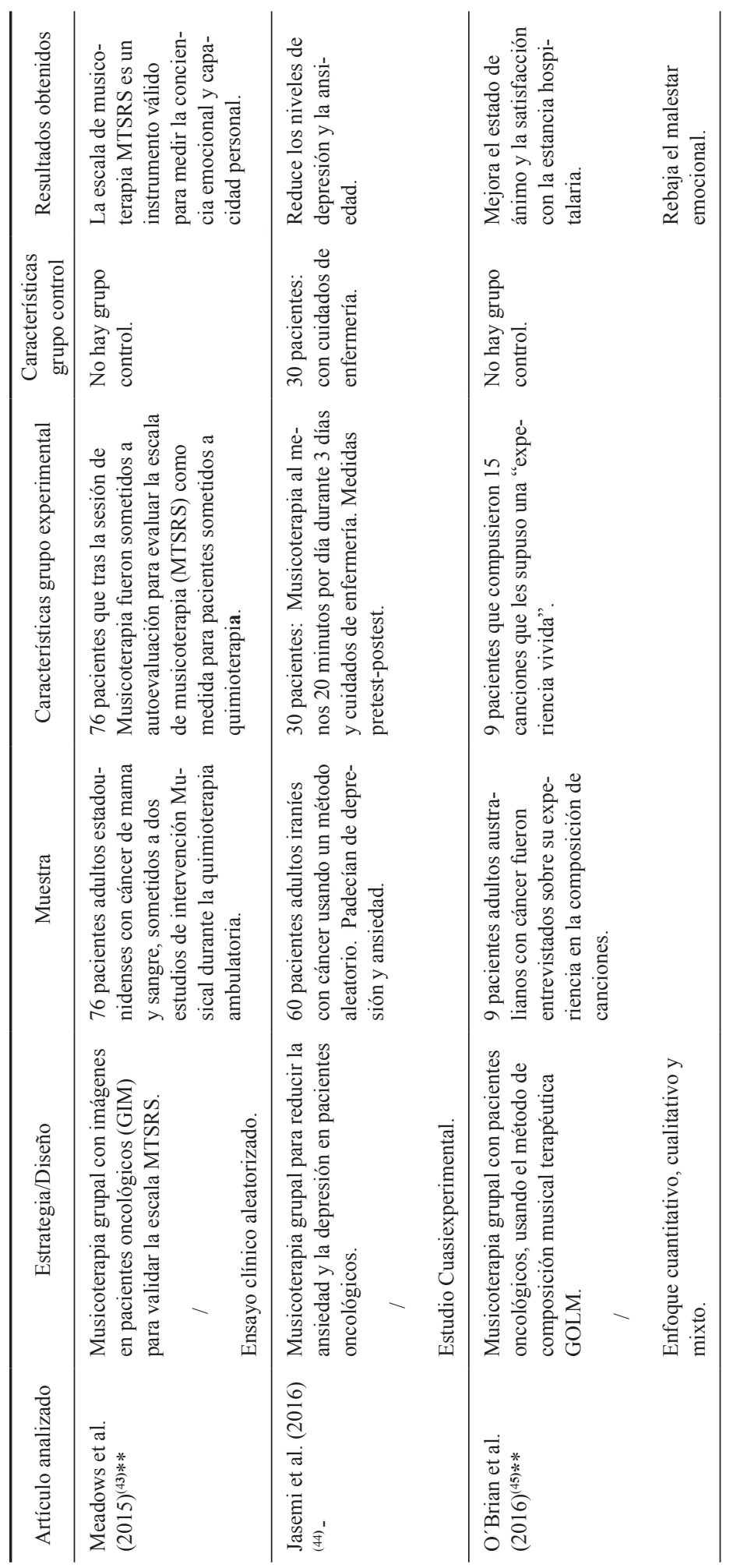




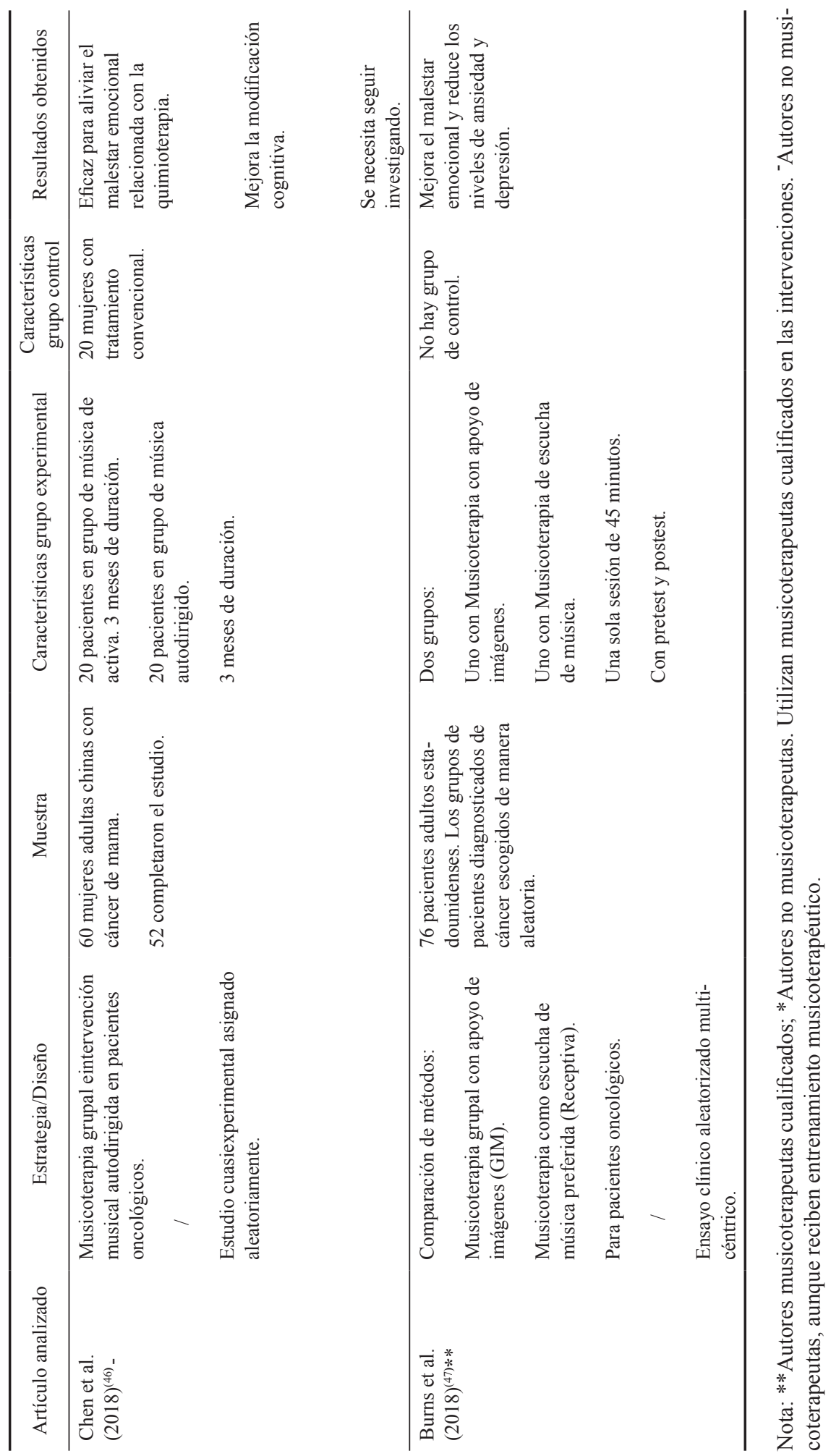




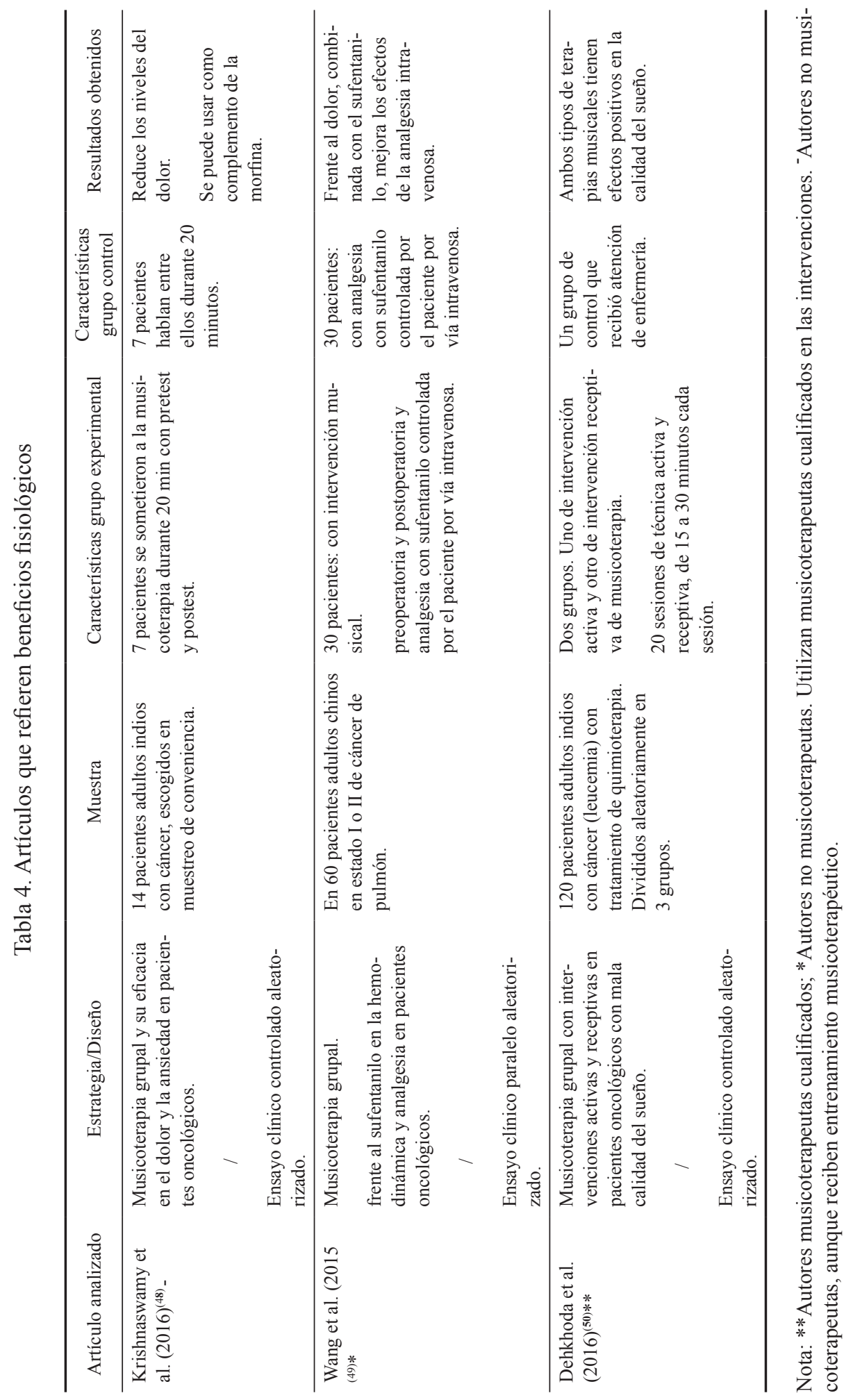




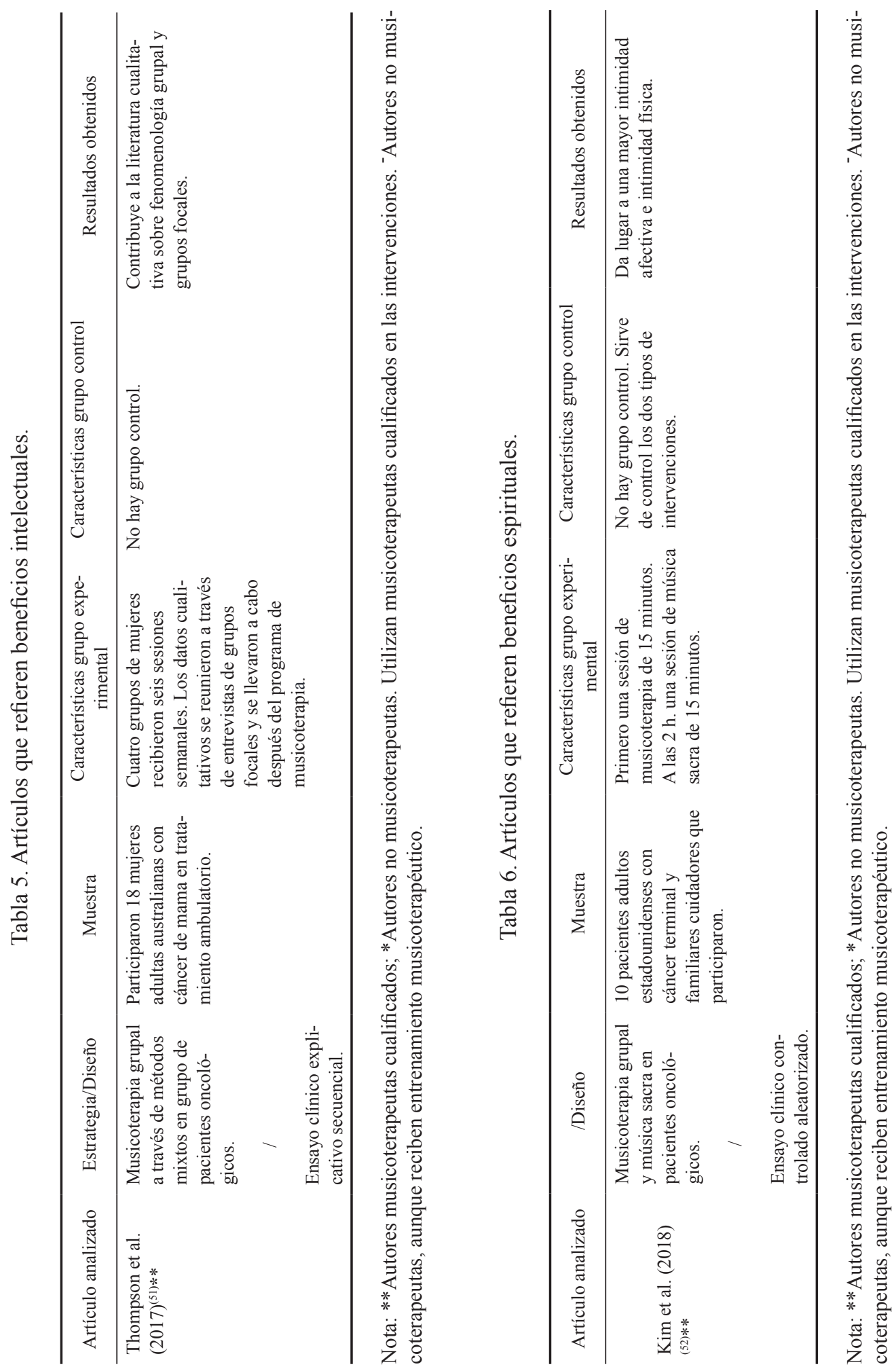


En la Tabla 3 se recogen nueve artículos sobre los beneficios psicológicos manifestados en pacientes con cáncer. De ellos, cuatro aplicados a mujeres adultas españolas con cáncer de mama, que demuestran una mejora del estado anímico, reduciendo la ansiedad y la depresión y mejorando la calidad de vida. Un artículo con adultos españoles con cáncer colorrectal que demuestran una reducción de la ansiedad y la depresión, al igual que otros cuatro artículos que lo refieren en pacientes con cáncer, sin especificar. Por último, un artículo hace referencia a niños mejicanos con cáncer que no obtienen beneficios en el manejo de la ansiedad, mientras sus cuidadores si los demuestran.

En la Tabla 4 se recopilan tres artículos que refieren beneficios fisiológicos en pacientes indios relacionados con la reducción de los niveles del dolor, pudiéndose usar como complemento de la morfina. En pacientes chinos con cáncer de pulmón reduce el dolor y, combinada con el sufentanilo, mejora los efectos de la analgesia intravenosa. En pacientes adultos indios con cáncer y con leucemia, la música activa y receptiva tiene efectos positivos en la calidad del sueño.

En la Tabla 5 se recoge un artículo que refiere beneficios intelectuales en pacientes adultas australianas con cáncer de mama y demuestra la importancia de los debates, en grupo focal, tras las sesiones de musicoterapia.

En la Tabla 6, se muestra un artículo que menciona beneficios espirituales en pacientes adultos con cáncer terminal que, a través de la musicoterapia, consiguen consuelo y trascendencia espiritual.

\section{Resultados}

Observando los datos expuestos anteriormente y en relación a la patología objeto de estudio, corresponde un artículo referido a pacientes con cáncer de mama y con cáncer de sangre (leucemia) de modo simultáneo $(5,88 \%)$, cinco artículos al cáncer de mama $(29,41 \%)$, cinco a cáncer no identificado en la investigación $(29,41 \%)$, uno al cáncer de colón $(5,88 \%)$, uno al cáncer de sangre $(5,88 \%)$, uno en cuidados paliativos $(5,88 \%)$, uno al de niños con cáncer y sus cuidadores $(5,88 \%)$, uno con cáncer terminal $(5,88 \%)$, uno al cáncer de pulmón $(5,88 \%)$. Fueron mayoría las intervenciones grupales $(94,11 \%)$ frente a las intervenciones individuales $(5,88 \%)$.

En cuanto a las franjas de edades, se evidencia que las propuestas van dirigidas a personas adultas $(94,11 \%)$, en su mayor parte dedicadas a un perfil de población femenina con cáncer de mama $(35,29 \%)$, y a niños y niñas y sus cuidadores $(5,88 \%)$.

En referencia a los países en los que se han realizado dichos tratamientos con musicoterapia y el estudio sobre los beneficios que reporta, destaca USA con cinco $(29,41 \%)$, China con tres $(17,64 \%)$, Australia, India y España con dos $(11,76 \%)$ y con uno (5,88\%) Méjico, Alemania e Irán.

Los diseños de investigación utilizados son muy variados y van desde el ensayo clínico controlado aleatorizado en cuatro $(23,52 \%)$, el aleatorizado de dos grupos en uno $(5,88 \%)$, el aleatorizado en uno $(5,88 \%)$, el controlado no aleatorio con grupo intervención y testigo en uno $(5,88 \%)$, el experimental intrasujeto de medidas repetidas, dos $(11,76 \%)$, el cruzado aleatorio en uno $(5,88 \%)$, el estudio cuasiexperimental en dos $(11,76 \%)$, el aleatorizado multicéntrico en uno $(5,88 \%)$, el paralelo aleatorizado en dos $(11,76 \%)$, el diseño explicativo secuencial en uno $(5,88 \%)$ hasta, finalmente, llegar al enfoque mixto en uno $(5,88 \%)$. 
A los pacientes asignados al grupo experimental se les aplicaron, además de la terapia convencional, intervenciones de musicoterapia. En diez de los artículos se detallan de manera genérica las técnicas utilizadas $(58,82 \%)$, pero en siete de ellos en los que no se especifican $(41,18 \%$ ) aunque sí hablan de la duración de las sesiones y los días que se invirtieron. Los cuidadores y/o familiares reciben sesiones de musicoterapia. En todos menos en uno la intervención fue grupal, lo que supone un $(94,12 \%)$ del total.

Las estrategias musicoterapéuticas más aplicadas fueron: música en directo y ejercicios de relajación; música variada al unísono con el Mindfulness, música receptiva, musicoterapia con métodos mixtos (asociada a las técnicas de relajación); musicoterapia con música grabada (generalmente clásica, con uso de imágenes); composición de canciones y música interactiva para la relajación; intervención musical autodirigida, y el uso de la musicoterapia en combinación con música sacra.

Las intervenciones son llevadas a cabo por musicoterapeutas de profesión. En nueve de ellos $(52,94 \%)$ uno de los autores está cualificado. En uno $(5,88 \%)$ utilizan profesionales cualificados y en cinco $(29,41 \%)$ ninguno de los autores posee dicha titulación, aunque previamente han recibido entrenamiento para llevarla a cabo.

Del análisis de los modelos de Musicoterapia utilizados, se desprende que en cinco de ellos $(29,41 \%)$ no se especifican las técnicas utilizadas de manera formal o son de libre configuración -el musicoterapeuta no sigue un patrón oficial y utiliza técnicas de diferentes modelos-. En otros cinco trabajos $(29,41 \%)$ la musicoterapia es aplicada al unísono con técnicas de relajación. Se aplican métodos mixtos con música activa y música receptiva en tres (17,64\%); el Guided Imagery and Music (GIM) en dos (11,79\%); el Modelo Alvin de improvisación, composición y análisis, en uno $(5,88 \%)$ y uso de musicoterapia alternada con música sacra en uno $(5,88 \%)$.

De los seis modelos diferentes de intervención de musicoterapia empleados, se derivan los siguientes beneficios:

- En tres artículos se encontraron beneficios psicológicos y fisiológicos. En el primero participan 84 pacientes de cáncer adultos, alemanes, hospitalizados en cuidados paliativos. Usando un ensayo controlado aleatorizado participan 42 pacientes en el grupo experimental al que se le aplican técnicas de musicoterapia con técnicas de relajación muscular y 42 en el grupo control que escucharon un ejercicio de relajación verbal. Los resultados refieren beneficios psicológicos en el grupo experimental, según Warth et al. $(2015)^{(37)}$, porque la musicoterapia influye en el bienestar de las personas con enfermedades terminales sometidas a cuidados paliativos y beneficios fisiológicos influyendo en la relajación. En el siguiente participan 15 mujeres adultas estadounidenses con cáncer de mama que recibieron sesiones de musicoterapia utilizada simultáneamente con el mindfulness. No hay grupo control. Usando un ensayo clínico controlado aleatorizado pretest-postest, los 15 pacientes recibieron sesiones de musicoterapia individualmente durante una hora por semana a lo largo de cuatro semanas. Se derivan beneficios psicológicos mejorando significativamente el estado de ánimo y beneficios fisiológicos disminuyendo el estado de fatiga, informado por Lesiuk $(2015)^{(38)}$. En el tercero participaron 31 pacientes estadounidenses oncológicos. Usando un ensayo clínico cruzado aleatorio pretest-potest sin grupo de control, recibieron dos sesiones usando música 
interactiva y dos sesiones en las cuales escucharon música pregrabada sin la presencia de un terapeuta (Brad et al., 2015) ${ }^{(42)}$. La información recabada por Martí et al. $(2015 \mathrm{c})^{(40)}$ identifica beneficios psicológicos de mejora del estado de ánimo, ansiedad y beneficios fisiológicos influyendo en la relajación y disminuyendo el dolor.

- En nueve artículos se encontraron beneficios psicológicos: en cuatro de ellos participaron mujeres con cáncer de mama, a las que se les aplican técnicas diferentes de musicoterapia, coincidiendo todos en la obtención de mejoras en el estado anímico, reduciendo la ansiedad y la depresión, informado por Martí et al.(2015c) $)^{(40)}$, y Zhou et al. (2015) el malestar emocional producida por la quimioterapia según Brad et al. $(2015)^{(42)}$ y mejorando la conciencia emocional, informado por Meadows et al. $(2015)^{(43)}$. En tres artículos participan pacientes en los que no se especifican los tipos de cáncer que padecían y a los que se les aplican distintas técnicas musicoterapéuticas. Jasemi et al. (2016) ${ }^{(44)}$ y O'Brian et al. $(2016)^{(45)}$, constatan disminución de la depresión y la ansiedad, y Chen et al. (2018) ${ }^{(46)}$, encuentran que rebaja el malestar emocional, obteniendo una mayor satisfacción con la estancia hospitalaria. En otro participaron 18 pacientes españoles (14 mujeres y 4 hombres) con cáncer colorrectal a los que, mediante un diseño experimental intrasujeto de medidas repetidas, se le aplicaron 8 sesiones de Musicoterapia, percibiendo una reducción de la ansiedad y la depresión y percibiendo una mejora de la calidad de vida, información recabada por Martí et al. $(2015 b)^{(39)}$. En el último artículo encontrado, informado por Cabral et al. (2014) ${ }^{(9)}$, participaron 112 pacientes pediátricos oncológicos mejicanos y 128 cuidadores en un ensayo clínico musicoterapéutico controlado no aleatorio con grupo de intervención y testigo, que produjo una disminución de la ansiedad en los cuidadores, pero no así en los pacientes.

- En tres artículos se encontraron beneficios fisiológicos: en uno de ellos participaron 14 pacientes oncológicos hindúes. Se usa un ensayo clínico controlado aleatorizado. En el grupo experimental participaron 7 pacientes que se sometieron a musicoterapia durante 20 minutos con pretest y postest y en el grupo control participan 7 pacientes que hablan entre ellos durante 20 minutos. La información facilitada por Krishnaswamy et al. (2016) ${ }^{(48)}$ refiere que los pacientes del grupo experimental manifiestan beneficios fisiológicos y que la musicoterapia puede ser usada como coadyuvante en la analgesia del dolor. En un segundo participaron 60 pacientes adultos chinos en estado I o II de cáncer de pulmón. Se usa un ensayo clínico paralelo aleatorizado. En el grupo experimental participaron 30 pacientes a los que se les aplica musicoterapia en el preoperatorio y en el postoperatorio y analgesia con sufentanilo. En el grupo control participaron 30 pacientes con solo analgesia con sufentanilo. Descubren que los y las participantes en el grupo experimental obtienen beneficios fisiológicos y que según Wang et al. (2015) $)^{(49)}$, la musicoterapia puede ser usada como coadyuvante en la analgesia del dolor. En el tercero participaron 120 pacientes adultos hindúes con cáncer (leucemia) en tratamiento de quimioterapia, divididos 
en tres grupos. Se usa un ensayo clínico controlado aleatorizado. Se forman dos grupos en el grupo experimental, uno de intervención musicoterapéutica activa y otro de intervención receptiva. Se aplicaron 20 sesiones con cada una de las técnicas (activas y receptivas) con una duración aproximada de 15 a 30 minutos cada sesión. En el grupo control se aplica ayuda de enfermería. Encuentran que los y las participantes del grupo experimental que reciben ambos tipos de terapias musicales (activas y receptivas) obtienen beneficios fisiológicos con efectos positivos en la calidad del sueño (Dehkhoda et al., 2016) ${ }^{(50)}$.

- En un artículo se encontraron beneficios intelectuales. En él participaron 18 mujeres adultas australianas con cáncer de mama en tratamiento ambulatorio. Se usa un ensayo clínico explicativo secuencial sin grupo control. En el grupo experimental se formaron cuatro grupos de mujeres que recibieron seis sesiones de musicoterapia semanales. Los datos cualitativos se reunieron a través de entrevistas de grupos focales y se llevaron a cabo después del programa de musicoterapia. Se concluye, según Thompson et al. $(2017)^{(51)}$, que la musicoterapia contribuye a la literatura cualitativa sobre fenomenología grupal y grupos focales.

- Finalmente, en un artículo se encontraron beneficios espirituales. Participaron 10 pacientes adultos estadounidenses con cáncer terminal y familiares cuidadores. Se usa un ensayo clínico controlado aleatorizado. Los y las participantes reciben musicoterapia alternando con música sacra. Kim et al. (2018) $)^{(52)}$, refieren beneficios espirituales dando lugar a una mayor intimidad afectiva y física y consuelo espiritual.

\section{Conclusiones}

Teniendo en cuenta que el objetivo de este estudio ha supuesto identificar la eficacia de los tratamientos musicoterapéuticos que aborden la sintomatología en pacientes con cáncer y que evidencien algún tipo de beneficio, el trabajo empírico realizado demuestra que la Musicoterapia es válida como opción no-farmacológica, en los siguientes términos:

Aporta beneficios fisiológicos por su influencia positiva en la relajación en pacientes hospitalizados en cuidados paliativos ${ }^{(46)}$, beneficios en consonancia con lo apuntado por Díaz et al. ${ }^{(30)}$.

Proporciona beneficios psicológicos en enfermas con cáncer de mama, disminuyendo ansiedad, estrés y depresión y mejorando su calidad de vida, información recabada por Martí et al. ${ }^{(39,40)}$; aliviando el malestar emocional relacionada con la quimioterapia y mejorando su $\operatorname{cognición}^{(41)}$, evidencias que coinciden con lo señalado por Martí et al. ${ }^{(12)} \mathrm{y}$, consecuentemente, ayudando a restablecer el equilibrio mental del individuo portador de trastornos mentales y funciones cognitivas y fisiológicas, como apuntaran Marins et al. ${ }^{(18)}$. Reporta beneficios intelectuales, demostrando la importancia de los debates, en grupo focal, tras las sesiones de musicoterapia ${ }^{(51)}$ y potencia los beneficios espirituales, al detectarse mayor intimidad afectiva y física en familiares de enfermos con cáncer terminal ${ }^{(52)}$, resultados en la línea de lo obtenido por Foster ${ }^{(34)}$. 
Con estos resultados sobre los beneficios para la salud, se debiera otorgar el alto valor que le corresponde a la musicoterapia como una terapia dinámica e integradora. Es el musicoterapeuta un especialista que, junto a equipos interdisciplinares de la medicina, ayuda a los pacientes, a sus familiares y/o cuidadores a superar las manifestaciones negativas del cáncer, al aplicar la música con una finalidad terapéutica.

En definitiva, la Musicoterapia hace que el paciente exprese sus subjetividades y establezca una relación entre paciente y musicoterapeuta que haga que se minimicen los efectos fisiológicos de tratamientos específicos oncológicos como la quimioterapia, o mejora de la ansiedad y de la depresión, se use como complemento de la morfina o el sufentanilo para que disminuya el dolor y otros tantos beneficios que resultan de especial importancia para estos pacientes. Como se ha podido ver, la Musicoterapia se muestra efectiva como terapia alternativa integrativa y complementaria al tratamiento convencional, intentando dar una solución nofarmacológica sin efectos secundarios.

\section{Referencias bibliográficas}

1. Martí P, Mercadal, M. Solé, C. La musicoterapia en Oncología. Gac Mex Oncolog 2015; 14: 346-52. doi:10.1016/j.gamo.2015.11.013

2. De Prados C, Viteri A, Fuente N. Aspectos psicológicos del paciente con cáncer. Gac Méd Bilbao 2003; 100: 28-31. [acceso el 10 de marzo de 2020]. Disponible en: http:// www.gacetamedicabilbao.eus/index.php/gacetamedicabilbao/article/viewFile/535/543

3. Bradt J, Dileo C, Magill L, Teague A. Music interventions for improving psychological and physical outcomes in cancer patients (Review). Cochrane Database Syst Rev 2016;(8):CD006911. doi:10.1002/14651858.CD006911.pub3

4. Quiroga M, Mallea C, Acevedo S. Terapias complementarias: camino hacia la oncología integrativa. Contac Científ 2016; [acceso el 11 de marzo de 2020]. 5: 37-42. Disponible en: http://contactocientifico.alemana.cl/ojs/index.php/cc/article/view/291

5. Podda RA., Sabbatella P, Suvini F. Musicoterapia en oncohematología infantil y pediátrica: interacción formativa, clínica y musical. Actas del VII Congreso Nacional de Musicoterapia. [Internet]. Segovia 2019. [acceso el 12 de marzo de 2020]. Disponible en: http://www.congresonacionaldemusicoterapia.es/wp-content/uploads/2019/10/ TEXTO-RESUMEN-TOTAL.PDF.pdf

6. Romero M, Mompe FJ. Musicoterapia en ASPANOA en la planta de oncopediatría del hospital infantil Miguel Servet de Zaragoza. Actas del V Congreso Nacional de Musicoterapia. [Internet]. Barcelona 17-19 de octubre de 2014. [acceso el 12 de marzo de 2020]. Disponible en: https://www.geyseco.es/musicoterapia/documentos/Libro_Ac tas.pdf

7. Sánchez O. Musicoterapia hospitalaria: cuidando con la música y el silencio en oncología pediátrica. Actas del VII Congreso Nacional de Musicoterapia. [Internet]. Segovia 2019. [acceso el 13 de marzo de 2020]. Disponible en: http://www.congresonacionaldemusi coterapia.es/wp-content/uploads/2019/10/TEXTO-RESUMEN-TOTAL.PDF.pdf 
8. Vaillancourt G. Música y Musicoterapia. Su importancia en el desarrollo infantil. Madrid: Editorial Narcea. 2009.

9. Cabral MC, Delgadillo AO, Flores EM, Sánchez FA. Manejo de la ansiedad en el paciente pediátrico oncológico y su cuidador durante la hospitalización a través de Musicoterapia. Psicooncología 2014: 11: 243-58. doi:10.5209/rev_PSIC. 2014.v11.n23.47386

10. American Music Therapy Association. Music Therapy and Military Populations. Editado AMTA, 2014. [acceso el 21 de marzo de 2020]. pp. 1-44. Disponible en: http:// www.musictherapy.org/assets/1/7/MusicTherapyMilitaryPops_2014.pdf

11. Poch S. Musicoterapia 2002. Programa de formación para mediadores en Musicoterapia y discapacidad. Libro de ponencias. [Internet] Madrid: Editan ASPACE, FEISD, Federación autismo de España. 2002. [acceso el 11 de marzo de 2020]. Disponible en: https://sid.usal.es/idocs/F8/FDO6823/musicoterapia.pdf

12. Lacárcel J. Psicología de la Música y de la emoción musical. Rev Educatio 2003. [acceso el 21 de marzo de 2020]. 20: 213-26. Disponible en: https://revistas.um.es/ educatio/article/view/138

13. Ready T. Music as language. Amer Jou Hosp Palliat Med 2010; 27: 7-15.. doi:10.1177/1049909109338387

14. Benedito MC. Reflexiones en torno a la utilidad de la música en la terapia psicológica con adolescentes. Rev Esp Pediatr 2010. [acceso el 22 de marzo de 2020]. 66: 136-40. Disponible en: https://www.seinap.es/wp-content/uploads/Revista-de-Pediatria/2010/ REP\%2066-2.pdf\#page $=56$

15. Oslé R. Musicoterapia y Psicoterapia. Rev Avanc Salud Ment Relac 2011. [acceso el 22 de marzo de 2020]. 10: 1-10. Disponible en: https://psiquiatria.com/bibliopsiquis/ musicoterapia-y-psicoterapia

16. Ribeiro KA., Alcântara RM., Oliveira CM., Paula TC., Dutra BR., Pedrino GR, Rebelo CS. Music therapy intervention in cardiac autonomic modulation, anxiety, and depression in mothers of preterms: Randomized controlled trial. BMC Psychol 2018; 1-10. doi:10.1186/s40359-018-0271-y

17. Fernández, M. E., Liébana, C. y López, C. La música como cuidado enfermero: Revisión sistemática. Rev Rol de Enferm 2018; [41: 28-35 [Acceso el 23 de marzo de 2020].. Disponible en: https://medes.com/publication/141807

18. Marins V, Rangel E, de Nazareth AB, Braga LD, Pereira D, Azevedo AS. Music Therapy in Patients with Mental Disorders. Journal of Nursing UFPE / Rev Enferm UFPE 2018. [acceso el 24 de marzo de 2020]. 12: 1054-9. Disponible en: https://periodicos.ufpe.br/ revistas/revistaenfermagem/article/view/231436/28676

19. Millán JC, Guillén P, Mayán JM. La Musicoterapia como técnica de desarrollo psicomotriz en la tercera edad. GEROKOMOS 1994. [Acceso el 2 de abril de 2020]. 5: 59-64. Disponible en: https://ruc.udc.es/dspace/bitstream/handle/2183/14324/ MillanCalenti_Musicoterapia.pdf

20. Gold C, Wigram T, Elefant C. Musicoterapia para el trastorno de espectro autista (Revisión Cochrane traducida). Bibliot Cochrane Plus 2008; 2: 1-22. [acceso el 28 de marzo de 2020]. Disponible en: http://www.sld.cu/galerias/pdf/sitios/mednat/ musicoterapia_para_el_trastorno_de_espectro_autista.pdf

21. Gattino G, Elefant C, Geretsegger M, Mössler K, Suvini F, Odell-Miller H, Watts G. The good, bad and ugly: joys and challenges of being involved in international research with children with autism. Nord J Music Ther 2016; 25: 89-89. doi:10.1080/08098131 .2016.1180084 
22. Marom M K, Gilboa A, Bodner E. Musical features and interactional functions of echolalia in children with autism within the music therapy dyad. Nordic J Music Ther 2018;27: 175-96. doi:10.1080/08098131.2017.1403948

23. Bergmann T, Sappok T, Diefenbacher A, Dziobek I. Music in diagnostics: using musical interactional settings for diagnosing autism in adults with intellectual developmental disabilities. Nord J Music Ther 2016; 25: 319-51. doi:10.1080/08098131.2015.1039567

24. Valdés, B., Martínez, I., Cuellar, L., Núñez, P. y Moreno, Y. Musicoterapia en niños con Trastorno por déficit de atención con Hipercinesia. X Sem Intern Atenc Prim Salud, Versión Virtual 2012. [Internet]. [acceso el 28 de marzo de 2020]. pp.1-9. Disponible en: http://cimfcuba2012.sld.cu/index.php/xseminarioAPS/2012/paper/view/700/319

25. Nyklíček I. Chapter 7. Mindfulness, emotion regulation, and well-being. Springer New York. In Emotion regulation and well-being 2011. [Acceso el 22 de marzo de 2020]. pp. 101-118. Disponible en: http://www.mariskakret.com/wp-content/uploads/2015/10/ Kret_Springer_2010.pdf\#page $=118$

26. Orjuela JM. Efecto ansiolítico de la Musicoterapia: aspectos neurobiológicos y cognoscitivos del procesamiento musical. Rev Colomb Psiquiat 2011; 40: 748-58 [Acceso el 23 de marzo de 2020].. Disponible en https://www.redalyc.org/pdf/806/80622 345011.pdf

27. Montalbán S. ¿Una sesión de musicoterapia reduce el dolor de los pacientes de cuidados paliativos? Evidentia 2014; [acceso el 23 de marzo de 2020]. 11: 47-8. Disponible en: https://dialnet.unirioja.es/servlet/articulo?codigo $=5248207$

28. Osborne, N. Neuroscience and "real world" practice: music as a therapeutic resource for children in zones of conflict. Ann N Y Acad Sci 2012; 1252:69-76. doi:10.1111 j.1749-6632.2012.06473. x.

29. Bernabé, M.M. Prácticas musicales para personas mayores: aprendizaje y terapia, Rev Facul Educ Albacete 2013. [acceso el 25 de marzo de 2020]. 28: 133-53. Disponible en http://www.revista.uclm.es/index.php/ ensayos

30. Díaz-Morales K, Reyes-Arvizu J, Morgado-Nájera K, Everardo-Domínguez D. Síntomas en niños con cáncer y estrategias de cuidado familiar. Rev Cuid 2019; 10: e597. doi:10.15649/cuidarte.v10i1.59

31. Moreira SV, dos Reis Justi FR, Moreira M. Can musical intervention improve memory in Alzheimer's patients? Evidence from a systematic review. Dementia Neuropsychol 2018; 12:133-42. doi:10.1590/1980-57642018dn12-020005

32. Silverman MJ. Effects of educational music therapy on illness management knowledge and mood state in acute psychiatric inpatients: a randomized three-group effectiveness study. Nord J Mus Therapy 2016; 25: 57-75. doi:10.1080/08098131.2015.1008559

33. Silverman MJ. Quantitative comparison of group-based music therapy experiences in an acute care adult mental health setting: A four-group cluster-randomized study. Nord J Music Ther 2019; 28: 41-59. doi:10.1080/08098131.2018.1542614

34. Foster B. Review of episodes of relationship completion through song: Case studies of music therapy research in palliative care. Canad J Music Ther 2010; 16: 192-5. [Acceso el 24 de marzo de 2020]. Disponible en: https://www.questia.com/read/1P3-2067208731 /episodes-of-relationship-completion-through-song

35. Araujo V, Frederico R, Ferreira NC, Ribeiro E, Paes MJ. Effects of sacred music on the spiritual well-being of bereaved relatives: a randomized clinical trial. Rev Esc Enferm USP 2017; 51:1-8. [Acceso el 24 de marzo de 2020]. Disponible en: http://scielo.isciii. es/ pdf/eg/v15n42/clinica4.pdf 
36. Latorre A, Del Rincón D, Arnal J. Bases metodológicas de la investigación educativa. Barcelona: Experiencia, 2003.

37. Warth M, Kebler J, Hillecke TK, Bardenheuer HJ. Music Therapy in palliative care. a randomized controlled trial to evaluate effects on relaxation. Deutsc Arzteb Int 2015; 112: 788-94. doi:10.3238/arztebl.2015.0788

38. Lesiuk T. The effect of Attention based on Music Care Therapy and humour in women receiving adjuvant breast cancer chemotherapy: A pilot study. Oncol Nurs Forum 2015; 42; 276-82. doi:10.1188/15.ONF.276-282

39. Martí P, Mercadal M, Solé C. Efecto de la musicoterapia en el estado de ánimo y calidad de vida de pacientes con cáncer colorrectal. Psicooncología 2015; 12: 259-82. doi:10.5209/rev_PSIC. 2015.v12.n2-3.51008

40. Martí P, Mercadal M, Solé C. Efecto de la musicoterapia en el estado anímico y calidad de vida de un grupo de mujeres supervivientes de cáncer de mama. Psicooncología 2015; 12: 105-28. doi:10.5209/rev_PSIC. 2015.v12.n1.48907

41. Zhou K, Li X, Li J, Liu M, Dang S, Xin X, et al. A clinical randomized controlled trial of music therapy and progressive muscle relaxation training in female breast cancer patients after radical mastectomy: results on depression, anxiety and length of hospital stay. Eur J Oncol Nurs 2015; 19: 54-9. doi:10.1016/j.ejon.2014.07.010

42. Bradt J, Potvin N, Kesslick A, Shim M, Radl D, Schriver E, et al. The impact of music therapy versus music medicine on psychological outcomes and pain in cancer patients: a mixed methods study. Support Care Cancer 2015; 23: 1261-71. doi:10.1007/s00520014-2478-7

43. Meadows A, Burns D, Perkins S. Measuring supportive music and imagery interventions: the development of the Music Therapy Self-Rating Scale. J Music Ther 2015, 52: 35375. doi:10.1093/jmt/thv010

44. Jasemi M, Aazami S, Zabihi RE. The effects of music therapy on anxiety and depression of cancer patients. Ind J Palliat Care 2016; 22: 455-8. doi:10.4103/0973-1075.191823

45. O'Brian E, Grocke D, Dileo C. I felt a bit a daunted, I've never written a song before: cancer patients' experiences of original song writing. Nord J Music Ther 2016; 25(sup1): 54. doi:10.1080/08098131.2016.1179966

46. Chen SC, Chou CC, Chang, H. J., \& Lin, M.-F. Comparison of group vs self-directed music interventions to reduce chemotherapy-related distress and cognitive appraisal: an exploratory study. Support Care Cancer 2018; 26: 461-9. doi:10.1007/s00520-0173850-1

47. Burns DS, Meadows AN, Althouse S, Perkins SM, Cripe LD. Differences between supportive music and imagery and music listening during outpatient chemotherapy and potential moderators of treatment effects. J Music Ther 2018; 55: 83-108. doi:10.1093/ jmt/thy001

48. Krishnaswamy, P, Nair S. Effect of music therapy on pain and anxiety levels of Cancer patients: a pilot study. Ind J Palliat Care 2016; 22: 307-11. doi:10.4103/09731075.185042

49. Wang Y, Tang H, Guo Q, Liu J, Liu X, Luo J, Yang W. Effects of intravenous patientcontrolled sufentanil analgesia and music therapy on pain and hemodynamics after surgery for lung cancer: a randomized parallel study. J Altern Complement Medic 2015; 21: 667-72. doi:10.1089/acm.2014.0310

50. Dehkhoda F, Vinayak S, Vinayak R. Clinical trial of effect of music-therapy on sleep quality in blood cancer patients. N J Music Ther 2016; 25: 124-5. doi:10.1080/080981 31.2016 .1180154 
51. Thompson S, Grocke D, Dileo C. The use of descriptive group phenomenology in a study of mixed methods to understand the experience of music therapy for women with breast cancer. Nord J Music Ther 2017; 26: 320-37. doi:10.1080/08098131.2016.1239 648

52. Kim B, Dvorak AL. Music therapy and intimacy behaviours of hospice family caregivers in South Korea: A randomized crossover clinical trial. Nord J Music Ther 2018, 27: 218-34. doi:10.1080/08098131.2018.1427783 